\title{
Effectiveness of aerobic exercise on upper limb function following breast cancer treatment: a systematic review and meta-analysis
}

\author{
Yuling Yang ${ }^{1}$, Danfeng Gu², Yuan Qian ${ }^{3}$, Huihong Wang ${ }^{4}$, Xiaoying Chai ${ }^{5}$ \\ ${ }^{1}$ Department of Breast Surgery, Affiliated Hospital of Jiangnan University, Wuxi, China; ${ }^{2}$ Nursing Department, Affiliated Hospital of Jiangnan \\ University, Wuxi, China; ${ }^{3}$ Department of Oncology, Affiliated Hospital of Jiangnan University, Wuxi, China; ${ }^{4}$ Outpatient Department, Affiliated \\ Hospital of Jiangnan University, Wuxi, China; ${ }^{5}$ Department of Endocrinology, Affiliated Hospital of Jiangnan University, Wuxi, China \\ Contributions: (I) Conception and design: Y Yang; (II) Administrative support: D Gu, H Wang; (III) Provision of study materials or patients: D Gu; (IV) \\ Collection and assembly of data: X Chai; (V) Data analysis and interpretation: Y Yang; (VI) Manuscript writing: All authors; (VII) Final approval of \\ manuscript: All authors. \\ Correspondence to: Danfeng Gu. Nursing Department, Affiliated Hospital of Jiangnan University, 1000 Hefeng Road, Binhu District, Wuxi 214123, \\ China. Email: 1308594635@qq.com.
}

\begin{abstract}
Background Aerobic exercise is currently considered to be an effective method of rehabilitation in breast cancer patients. Studies have shown that aerobic exercise after breast cancer surgery can improve upper limb function, cardiopulmonary function, and quality of life. Breast cancer rehabilitation guidelines encourage patients to actively participate in aerobic exercise to promote rehabilitation, the current study is to evaluate the effectiveness of aerobic exercise on upper limb muscle strength and range of motion (ROM) following breast cancer treatment.
\end{abstract}

Methods: Electronic databases (Cochrane Library, PubMed, Web of Science, EBSCO and Embase) were searched for randomized controlled trials (RCTs) published before September 1, 2019, using the search terms "aerobic exercise", "exercise", "breast cancer", "muscle strength", "strength", "flexibility" and "function". Grip strength and shoulder joint ROM were used to evaluate upper limb strength and upper limb flexibility respectively. All statistical tests were performed using RevMan5.3 software.

Results: Nine RCTs (421 patients) were included for analysis, with JBI scores ranging from 19 to 23, and bias grade B for all studies among which, there were six studies reported change in grip strength, and five studies reported change in shoulder joint ROM. Meta-analysis showed a statistically significant difference in shoulder flexion ROM ( $\mathrm{MD}=4.97,95 \% \mathrm{CI}$ : 0.47-9.46, $\mathrm{P}=0.03)$, shoulder abduction $\mathrm{ROM}(\mathrm{MD}=8.95$, 95\% CI: 0.99-16.91, $\mathrm{P}=0.03)$, shoulder internal rotation ROM ( $\mathrm{MD}=3.45,95 \% \mathrm{CI}: 1.80-5.09, \mathrm{P}<0.0001)$, shoulder external rotation $\mathrm{ROM}(\mathrm{MD}=7.69,95 \% \mathrm{CI}: 0.06-15.32, \mathrm{P}=0.05)$ between the intervention and control groups following completion of the aerobic exercise intervention, while there were no significant improvement with respect to grip strength and shoulder extension $\mathrm{ROM}(\mathrm{P}>0.05)$.

Conclusions: Aerobic exercise could improve shoulder joint ROM in breast cancer survivors, but shows no obvious effect on the improvement of upper limb strength.

Keywords: Breast cancer; aerobic exercise; muscle strength; range of motion (ROM); meta-analysis

Submitted Nov 06, 2020. Accepted for publication Feb 26, 2021.

doi: 10.21037/apm-20-2616

View this article at: http://dx.doi.org/10.21037/apm-20-2616

\section{Introduction}

The International Cancer Research Center of WHO estimates there are 1.67 million new cases and 525,000 deaths from breast cancer every year, making it the second- most common cancer globally, and the most common cancer in women (1). The annual economic burden of breast cancer in China is about 970 million RMB (2).

Aerobic exercise is currently considered to be an effective 
method of rehabilitation in breast cancer patients. Studies have shown that aerobic exercise after breast cancer surgery can improve upper limb function, cardiopulmonary function, and quality of life (3-5). Breast cancer rehabilitation guidelines encourage patients to actively participate in aerobic exercise to promote rehabilitation (6).

This study aimed to explore the specific impact of aerobic exercise on upper limb function in breast cancer survivors through evidence-based methods, and to provide recommendations for effective rehabilitation of breast cancer survivors. In this study, the term 'breast cancer patients' refers to breast cancer patients who have completed intensive treatment.

We present the following article in accordance with the PRISMA reporting checklist (available at http://dx.doi. org/10.21037/apm-20-2616).

\section{Methods}

\section{Search strategy and eligibility criteria}

Systematic review of Cochrane and JBI libraries was performed with subsequent meta-analysis. A preliminary search of relevant original papers in Chinese and English databases (such as CNKI, Wanfang Data, Cochrane Library, PubMed) was conducted to analyze titles and abstracts to determine appropriate key words for the literature search. A database search was then conducted in five databases (Cochrane Library, PubMed, Web of Science, EBSCO and Embase) using the search terms "aerobic exercise", "exercise", "breast cancer", "muscle strength", "strength", "flexibility" and "function". Articles published from database inception until September 2019 were included. Titles and abstracts were screened, with abstracts that met the inclusion criteria undergoing review of full text to determine eligibility. Reference lists of included articles were screened for relevant studies.

\section{Inclusion and exclusion criteria}

\section{Type of study}

Randomized controlled trials (RCTs) investigating change in upper limb strength and flexibility in breast cancer survivors were included.

\section{Inclusion and exclusion criteria}

Studies including patients over 18 years old who had been diagnosed with breast cancer by pathology and had completed intensive treatment for breast cancer (including surgery, chemotherapy and/or radiotherapy) were included. Studies were required to have an intervention involving organized aerobic exercise and a control group that participated in routine nursing or routine activities in the same period. Patients with recurrence or metastasis of disease, or ongoing treatment, were excluded.

\section{The outcome index}

\section{Muscle strength of upper limb}

Foreign scholars highlighted grip strength as an effective way to measure upper limb muscle strength (7). Standard grip dynamometry was used to measure patients' grip strength. The grip dynamometer handle was adjusted to an appropriate position before the measurement and measurements were made in kilograms $(\mathrm{kg})$.

\section{Range of motion of upper limb joints}

According to the guidelines from The American Medical Association, maximum ROM of shoulder flexion (8), extension, abduction, internal rotation and external rotation were measured with goniometry. This requires the patient to stand with their arms by their side, elbows straight, and palms facing inward, with the vertical line used as the stationary axis. Shoulder flexion ROM is measured by lifting the arm forward to maximum height, placing the goniometer axis at the humeral head and measuring the angle between the stationary arm (parallel with the trunk) and moving arm (midline of the humerus). Shoulder extension and abduction ROM are measured using the same axis, stationary and moving arms, with extension measured by reaching the arm back as far as possible, and abduction measured by reaching the arm up and out to the side to maximum height. Internal and external rotation ROM is measured by rotating the shoulder inwardly and outwardly.

\section{Data extraction}

Search results were exported to EndNote $\mathrm{X} 7$ and duplicates were removed. Two researchers exported the citations to Microsoft Excel and conducted the literature screening and selection independently. Divergent judgements were settled through discussion.

\section{Literature quality evaluation}

Quality of the included studies was evaluated using the JBI scoring criteria. This included assessment of study purpose, 


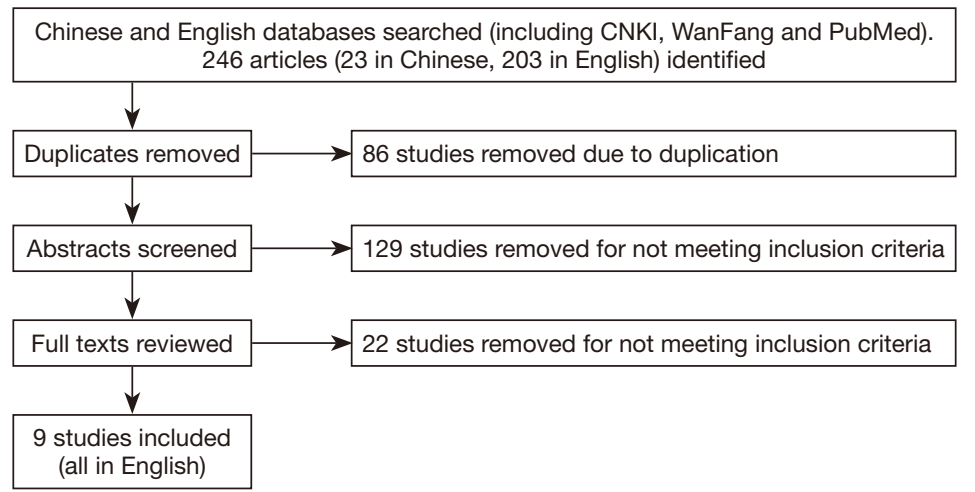

Figure 1 Literature screening process. Initial literature search yielded 246 potential studies for inclusion. After removing duplicate papers or papers that did not meet the inclusion criteria $(n=237), 9$ papers were included for analysis.

allocation method, blinding method, sample size, baseline comparison, description of loss to follow-up, research tools, data collection and follow-up method, statistical method, discussion of results, and references. The scoring criteria comprises 13 items, with a score of 0 denoting total failure to meet requirements, a score of 1 denoting partial fulfilment, and a score of 2 denoting complete fulfilment of requirements, for a total possible score of 26 points.

Bias grade was evaluated according to the Cochrane Handbook for Systematic Reviews of Interventions (9). Grade A states that a study "[met] all the criteria completely, with the least possibility of bias", grade B states that a study "[met] all the criteria partially, the probability of bias is moderate", and grade $\mathrm{C}$ states that a study "completely [does not meet] all the criteria, the probability of bias is high". Studies with a JBI score $>15$ and bias grade A or B were included.

\section{Data analysis method}

RevMan5.3 software was used for data meta-analysis with data imported from the included studies. Data was assessed for heterogeneity: if heterogeneity results were $\mathrm{I}^{2} \leq 50 \%$ and $\mathrm{P} \geq 0.1$, a fixed effects model was used for analysis; if $\mathrm{I}^{2}>50 \%, \mathrm{P}<0.1$, a random effects model was used. Effects values were combined and presented in a forest plot. All results were continuous variable data and mean difference was used to measure effect sizes. All effects were expressed as $95 \%$ confidence intervals (CIs) where $\mathrm{P}<0.05$ was considered statistically significant. Where the heterogeneity test was statistically significant, subgroup analysis was conducted to analyse factors that may have contributed to heterogeneity.

\section{Results}

\section{The results of the literature research}

Initial literature search yielded 246 potential studies for inclusion. After removing duplicate papers or papers that did not meet the inclusion criteria $(n=237), 9$ papers were included for analysis (Figure 1).

\section{Methodological quality of included studies}

Nine RCTs were included, with JBI scores ranging from 19 to 23 , and bias grade B for all studies, indicating high quality of included studies.

\section{Methods of aerobic exercise}

All studies included in this meta-analysis used exercise methods adopted in the intervention group included Aerobics Pulse, Tai Chi, and cycling. Intervention occurred after intensive breast cancer treatment. The longitudinal duration of the intervention ranged from 42 days to 12 months, with each episode of aerobic exercise lasting from 40 to 60 minutes each. Eight studies used professional instructors to guide and supervise patients through a standardized exercise regime, and three studies outlined clear requirements and measures for evaluating exercise intensity. Table 1 provides an overview of the included studies.

\section{Effects of aerobic exercise on upper limb function in breast cancer patients}

Our meta-analysis evaluated upper limb function in patients based on grip strength and shoulder joint ROM. 
Heterogeneity analysis showed no heterogeneity, and a fixed effects model was used for meta-analysis.

Of the included studies, six evaluated change in grip strength at completion of the aerobic exercise program, which included 128 patients in the intervention group and 133 patients in the control group. Shoulder joint ROM was evaluated in five studies, which included 108 patients in the intervention group and 110 patients in the control group, with flexion, extension, abduction, internal rotation and external rotation being the main planes of movement recorded.

\section{Effects of aerobic exercise on grip strength in breast} cancer patients

After the completion of the aerobic exercise intervention program, no statistically significant difference was found in the grip strength between the intervention and control groups (MD $=5.52,95 \% \mathrm{CI}:-7.74-18.79, \mathrm{P}=0.41)$ (Figure 2).

Effects of aerobic exercise on shoulder ROM in breast cancer patients

Effects of aerobic exercise on the shoulder flexion ROM in breast cancer patients

Meta-analysis showed a statistically significant difference in shoulder flexion ROM between the intervention and control groups following completion of the aerobic exercise intervention $(\mathrm{MD}=4.97,95 \% \mathrm{CI}: 0.47-9.46, \mathrm{P}=0.03)$ (Figure 3).

\section{Effects of aerobic exercise on shoulder extension ROM in} breast cancer patients

Meta-analysis showed no statistically significant difference in shoulder extension ROM between the intervention and control groups following completion of the aerobic exercise intervention $(\mathrm{MD}=6.89,95 \% \mathrm{CI}:-4.77-18.55, \mathrm{P}=0.25)$ (Figure 4).

\section{Effects of aerobic exercise on shoulder abduction ROM in} breast cancer patients

Meta-analysis showed statistically significant difference in shoulder abduction ROM between the intervention and controls group following completion of the aerobic exercise intervention ( $\mathrm{MD}=8.95,95 \% \mathrm{CI}: 0.99-16.91, \mathrm{P}=0.03)$ (Figure 5).

Effects of aerobic exercise shoulder internal rotation ROM in breast cancer patients

Meta-analysis showed a statistically significant difference in shoulder internal rotation ROM between intervention and control groups following completion of the aerobic exercise intervention $(\mathrm{MD}=3.45,95 \% \mathrm{CI}: 1.80-5.09, \mathrm{P}<0.0001)$ (Figure 6).

Effects of aerobic exercise on shoulder external rotation ROM in breast cancer patients

Meta-analysis showed a statistically significant difference in shoulder external rotation ROM between intervention and control groups following completion of the aerobic exercise intervention ( $\mathrm{MD}=7.69,95 \% \mathrm{CI}: 0.06-15.32, \mathrm{P}=0.05)$ (Figure 7).

\section{Discussion}

Aerobic exercise is considered effective in promoting recovery in breast cancer patients following treatment, with advantages including its simplicity, economic-viability and highly-feasibility. Although studies suggest that aerobic exercise can improve upper limb function in breast cancer patients, there exist few systematic analyses targeting specific aspects of upper limb function.

The purpose of this study was to evaluate the effect of aerobic exercise on improving upper limb strength and shoulder ROM in breast cancer patients. All included RCTS compared intervention and control group data at baseline, with no significant differences between groups. All studies met inclusion criteria, with an intervention method of aerobic exercise, and control group of routine nursing or routine activities. JBI scores of included studies ranged from 19 to 23 , and bias was evaluated as grade B in all studies, indicating high quality and reliability.

The results of this review showed that aerobic training, even at high intensities, was feasible in a breast cancer population. There were no reports of adverse events caused by aerobic exercise in the included studies; however, training should still be conducted under professional guidance in a safe environment.

In contrast to Ibrahim's study (15), the results of this meta-analysis suggested that short-term exercise had no significant effect on improving grip strength in breast cancer patients, possibly due to the nature of the aerobic exercise intervention-which predominantly targeted core muscles rather than upper limb muscles-or the shorter intervention time.

Meta-analysis showed that aerobic exercise improved shoulder joint flexion, abduction, internal rotation and external rotation in breast cancer patients, but showed no significant effect on shoulder extension ROM. This could be due to movements of shoulder flexion, abduction, 


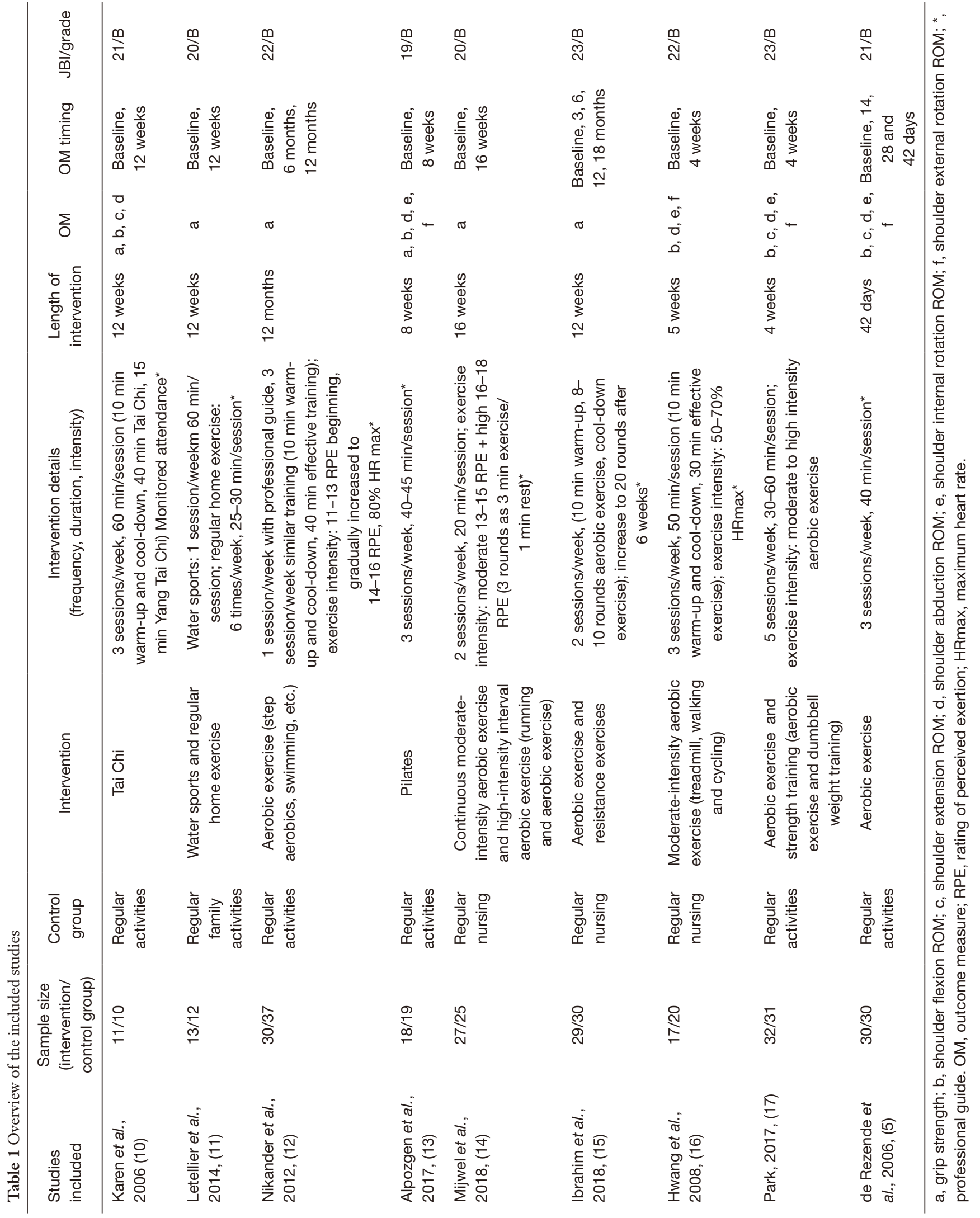




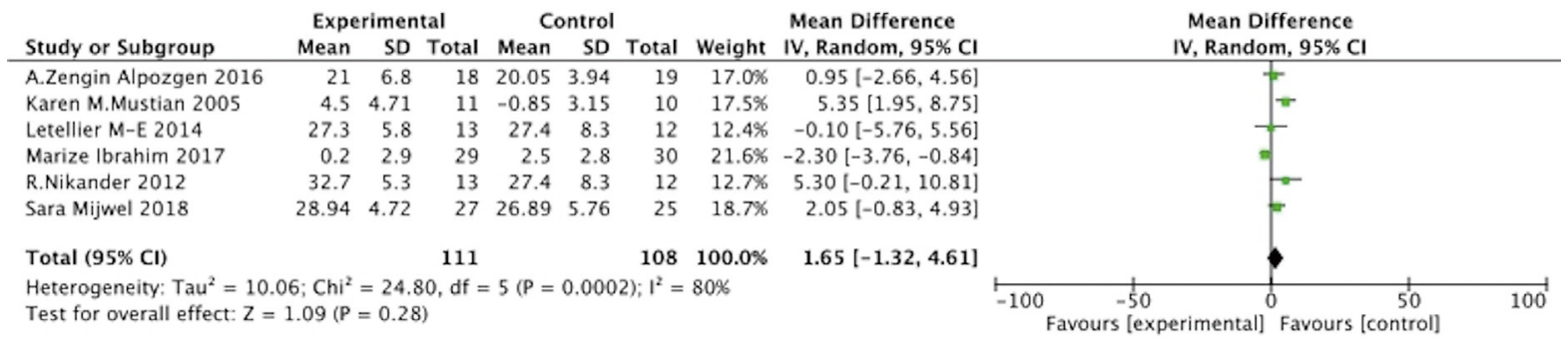

Figure 2 Effects of aerobic exercise on grip strength in breast cancer patients. After the completion of the aerobic exercise intervention program, no statistically significant difference was found in the grip strength between the intervention and control groups $(\mathrm{MD}=5.52,95 \%$ CI: -7.74-18.79, $\mathrm{P}=0.41)$. MD, mean deviation; $\mathrm{CI}$, confidence interval.

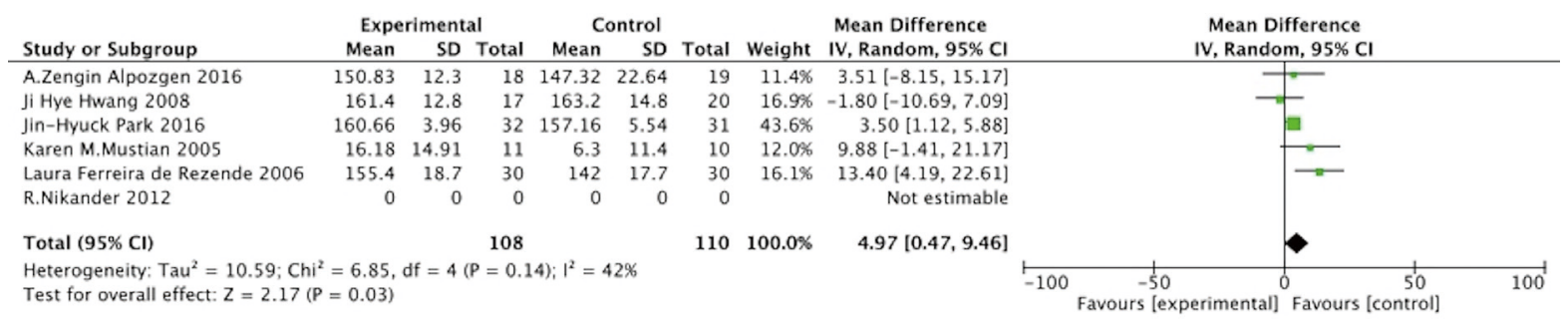

Figure 3 Effects of aerobic exercise on shoulder flexion ROM in breast cancer patients. Meta-analysis showed a statistically significant difference in shoulder flexion ROM between the intervention and control groups following completion of the aerobic exercise intervention ( $\mathrm{MD}=4.97,95 \% \mathrm{CI}: 0.47-9.46, \mathrm{P}=0.03)$. ROM, range of motion; MD, mean deviation; CI, confidence interval.

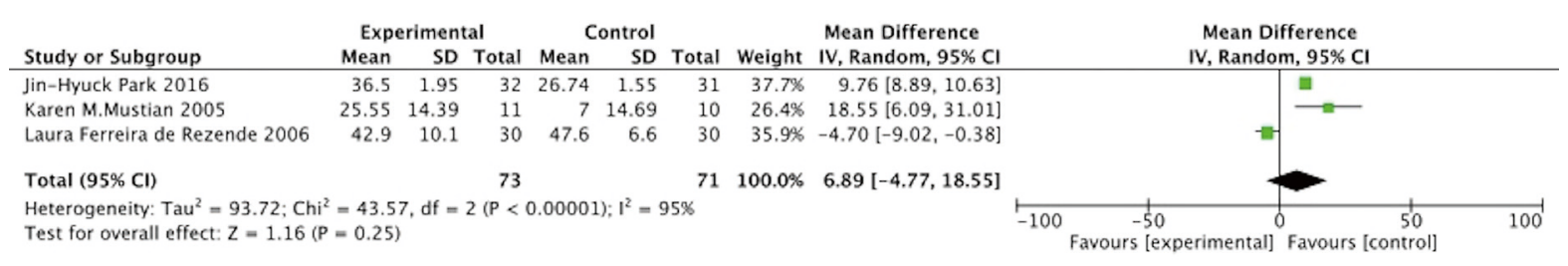

Figure 4 Effects of aerobic exercise on the shoulder extension ROM in breast cancer patients. Meta-analysis showed no statistically significant difference in shoulder extension ROM between the intervention and control groups following completion of the aerobic exercise intervention ( $\mathrm{MD}=6.89,95 \% \mathrm{CI}$ : $-4.77-18.55, \mathrm{P}=0.25)$. ROM, range of motion; $\mathrm{MD}$, mean deviation; CI, confidence interval.

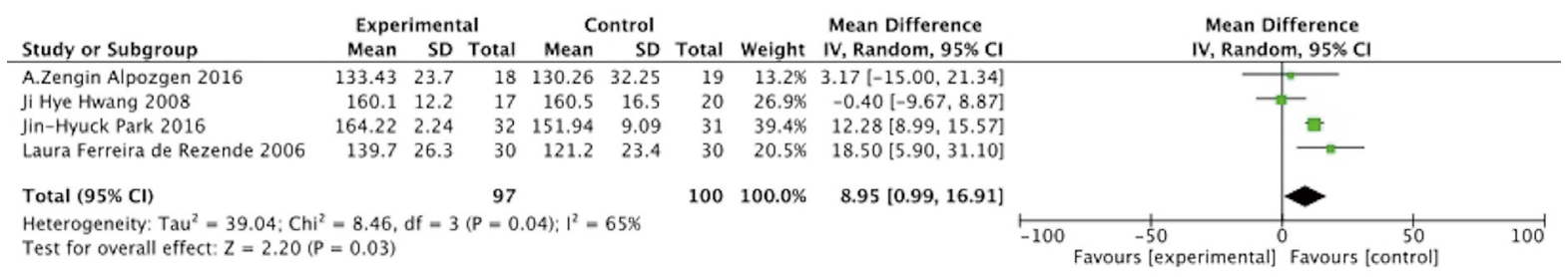

Figure 5 Effects of aerobic exercise on shoulder abduction ROM in breast cancer patients. Meta-analysis showed statistically significant difference in shoulder abduction ROM between the intervention and controls group following completion of the aerobic exercise intervention ( $\mathrm{MD}=8.95$, 95\% CI: 0.99-16.91, $\mathrm{P}=0.03$ ). ROM, range of motion; $\mathrm{MD}$, mean deviation; CI, confidence interval. 


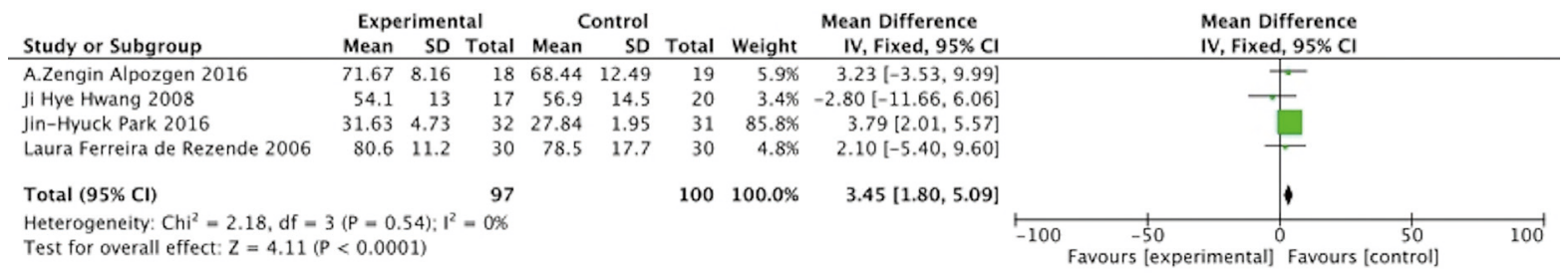

Figure 6 Effects of aerobic exercise on shoulder internal rotation ROM in breast cancer patients. Meta-analysis showed a statistically significant difference in shoulder internal rotation ROM between intervention and control groups following completion of the aerobic exercise intervention (MD =3.45, 95\% CI: 1.80-5.09, $\mathrm{P}<0.0001)$. ROM, range of motion; $\mathrm{MD}$, mean deviation; CI, confidence interval.

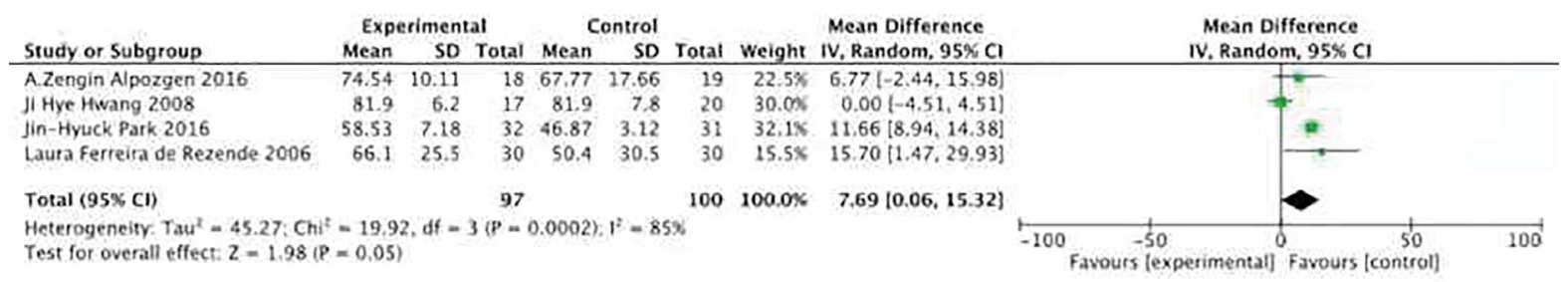

Figure 7 Effects of aerobic exercise on shoulder external rotation ROM in breast cancer patients. Meta-analysis showed a statistically significant difference in shoulder external rotation ROM between intervention and control groups following completion of the aerobic exercise intervention ( $\mathrm{MD}=7.69,95 \% \mathrm{CI}$ : 0.06-15.32, $\mathrm{P}=0.05$ ). ROM, range of motion; $\mathrm{MD}$, mean deviation; CI, confidence interval.

internal rotation and external rotation being more common in sports and day-to-day activities, while shoulder extension is a less functional, possibly leading to a lack of appreciable change following completion of the exercise program.

Aerobic exercise usually requires the participation of global muscle groups, which is conducive to improving cardiopulmonary status, limb function and balance. In this study, aerobic exercise was shown to effectively improve patients' shoulder joint ROM and, as such, should be widely promoted during the recovery period following breast cancer treatment. Aerobic exercise rehabilitation should be completed under professional guidance in a safe environment with a graded approach, in order to achieve improved recovery.

\section{Limitations of this study}

Due to the wide range of types and intensity of aerobic exercise, and the heterogeneity of intervention time and frequency, further subgroup analysis is suggested if large sample size is available. As this review only analysed published studies, there may be data from more recent and current trials that are not included with potential to impact these findings. Further studies that investigate specific exercise types and parameters are also needed.

\section{Conclusions}

Aerobic exercise significantly improves shoulder joint ROM following breast cancer treatment, but has no obvious effect on grip strength. It is suggested that patients recovering from breast cancer treatment should undertake regular aerobic exercise.

\section{Acknowledgments}

Funding: This article is funded by the Wuxi Municipal Health Commission, project number MS201945.

\section{Footnote}

Reporting Checklist: The authors have completed the PRISMA reporting checklist. Available at http://dx.doi. org/10.21037/apm-20-2616

Conflicts of Interest: All authors have completed the ICMJE uniform disclosure form (available at http://dx.doi. org/10.21037/apm-20-2616). The authors have no conflicts of interest to declare.

Ethical Statement: The authors are accountable for all 
aspects of the work in ensuring that questions related to the accuracy or integrity of any part of the work are appropriately investigated and resolved.

Open Access Statement: This is an Open Access article distributed in accordance with the Creative Commons Attribution-NonCommercial-NoDerivs 4.0 International License (CC BY-NC-ND 4.0), which permits the noncommercial replication and distribution of the article with the strict proviso that no changes or edits are made and the original work is properly cited (including links to both the formal publication through the relevant DOI and the license). See: https://creativecommons.org/licenses/by-nc-nd/4.0/.

\section{References}

1. Linghu R, Si W, Li Y, et al. Epidemiological and clinicopathological analysis of 3846 cases of breast cancer. Journal of PLA Medical College 2015;36:1017-38.

2. Wang L, Yue X, Shi J, et al. Research on the economic burden of breast cancer in China in the past 20 years. Prevention and Control of Chronic Diseases in China 2017;25:143-6.

3. Sandel SL, Judge JO, Landry N, et al. Dance and movement program improves quality-of-life measures in breast cancer survivors. Cancer Nurs2005;28:301-9.

4. Lahart IM, Carmichael AR, Nevill AM, et al. The effects of a home-based physical activity intervention on cardiorespiratory fitness in breast cancer survivors; a randomised controlled trial. J Sports Sci 2018;36:1077-86.

5. de Rezende LF, Franco RL, de Rezende MF, et al. Two exercise schemes in postoperative breast cancer: comparison of effects on shoulder movement and lymphatic disturbance. Tumori 2006;92:55-61.

6. Chinese Preventive Medical Association of Women's Health Branch of Mammary science Group. Lifestyle Guidelines for Breast cancer patients in China. Clinical Practice and Education in General Practice, 2017;15:124-8.

7. España-Romero V, Ortega FB, Vicente-Rodríguez G, et al. Elbow position affects handgrip strength in adolescents: validity and reliability of Jamar, DynEx, and TKK dynamometers. J Strength Cond Res 2010;24:272-7.

8. American Medical Association. Guides to the evaluation of permanent impairment. 4th ed. Chicago: American Medical Association; 1993.
9. Higgins J, Thomas J. Cochrane handbook for systematic reviews of interventions version 5.0. New Jersey: Wiley Blackwel, 2008:102-108.

10. Mustian KM, Katula JA, Zhao H. A pilot study to assess the influence of tai chi chuan on functional capacity among breast cancer survivors. J Support Oncol 2006;4:139-45.

11. Letellier ME, Towers A, Shimony A, et al. Breast cancerrelated lymphedema: a randomized controlled pilot and feasibility study. Am J Phys Med Rehabil 2014;93:751-9; quiz 760-1.

12. Nikander R, Sievänen H, Ojala K, et al. Effect of exercise on bone structural traits, physical performance and body composition in breast cancer patients--a 12-month RCT. J Musculoskelet Neuronal Interact 2012;12:127-35.

13. ZenginAlpozgen A, Razak Ozdincler A, Karanlik H, et al. Effectiveness of Pilates-based exercises on upper extremity disorders related with breast cancer treatment. Eur J Cancer Care (Engl) 2017;26. doi: 10.1111/ecc.12532.

14. Mijwel S, Backman M, Bolam KA, et al. Highly favorable physiological responses to concurrent resistance and high-intensity interval training during chemotherapy: the OptiTrain breast cancer trial. Breast Cancer Res Treat 2018;169:93-103.

15. Ibrahim M, Muanza T, Smirnow N, et al. A Pilot Randomized Controlled Trial on the Effects of a Progressive Exercise Program on the Range of Motion and Upper Extremity Grip Strength in Young Adults With Breast Cancer. Clin Breast Cancer 2018;18:e55-64.

16. Hwang JH, Chang HJ, Shim YH, et al. Effects of supervised exercise therapy in patients receiving radiotherapy for breast cancer. Yonsei Med J 2008;49:443-50.

17. Park JH. The effects of complex exercise on shoulder range of motion and pain for women with breast cancerrelated lymphedema: a single-blind, randomized controlled trial. Breast Cancer 2017;24:608-14.

(English Language Editor: M. Hawkins)

Cite this article as: Yang Y, Gu D, Qian Y, Wang H, Chai X. Effectiveness of aerobic exercise on upper limb function following breast cancer treatment: a systematic review and meta-analysis. Ann Palliat Med 2021;10(3):3396-3403. doi: 10.21037/apm-20-2616 